\title{
BMJ Open Lactate clearance as a useful biomarker for the prediction of all-cause mortality in critically ill patients: a systematic review study protocol
}

\author{
Zhongheng Zhang, Xiao Xu, Kun Chen
}

To cite: Zhang Z, Xu X, Chen K. Lactate clearance as a useful biomarker for the prediction of all-cause mortality in critically ill patients: a systematic review study protocol. BMJ Open 2014:4:e004752. doi:10.1136/bmjopen-2013004752

- Prepublication history for this paper is available online. To view these files please visit the journal online (http://dx.doi.org/10.1136/ bmjopen-2013-004752).

Received 28 December 2013 Revised 14 April 2014 Accepted 28 April 2014

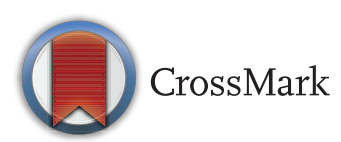

Department of Critical Care Medicine, Jinhua Municipal Central Hospital, Jinhua Hospital of Zhejiang University, Zhejiang, People's Republic of China

Correspondence to Dr Kun Chen; 13957970707@sina.com

\section{ABSTRACT \\ Introduction: Arterial lactate is a reflection of balance between lactate production and clearance. \\ Accumulating lactate may be a marker of global hypoxia or hypoperfusion. Lactate clearance is the reduction of lactate concentrations with interventional strategies, and it has been associated with increased risk of death in critically ill patients. However, conflicting results exist, which mandates a systematic review to clarify the association between lactate clearance and clinical outcome.}

Methods and analysis: Critically ill adult patients will be included in our analysis. This population will include heterogeneous study participants, including patients with sepsis or severe sepsis, trauma, surgical intensive care unit (ICU) patients, and so on. We will search four databases including EBSCO, PubMed, Scopus and ISI Web of knowledge from inception to February 2014. There will be no language restrictions in the electronic search for studies. Newcastle Ottawa Scale for cohort study will be employed to assess the reporting quality of included original studies. We will report pooled relative risk of death for those with lactate clearance and those without. The diagnostic performance of lactate clearance in predicting mortality will be explored by using the hierarchical summary receiver operating characteristic model.

Ethics and dissemination: The protocol for the systematic review has been registered in PROSPERO. The study will be disseminated electronically and in print. It will also be presented to conferences related to critical care medicine.

Trial registration number: CRD42013006511.

\section{INTRODUCTION}

Lactate is the end product of anaerobic glycolysis. In situations of hypoperfusion or hypoxia, pyruvate will no longer enter into the mitochondria for aerobic metabolism, but instead, it is preferentially reduced to lactate, resulting in accumulation of lactate in the blood. The process is an adaptive mechanism to produce energy in situations of low oxygen supply, but at the expense of deteriorating acidosis. ${ }^{12}$ Lactate is produced in the majority of tissues in human beings, but the main producers include red blood cells, intestine, brain and muscles. In critically ill patients, the daily production of lactate is approximately $1300 \mathrm{mmol} /$ day and the concentrations of arterial lactate represent the balance between production and clearance. Lactic acidosis or hyperlactatemia occurs when production exceeds clearance, which has been confirmed to be associated with worse clinical outcomes in critically ill patients. Even a mild increase in arterial lactate is associated with higher risk for worse outcome. Such association has been replicated in several patient populations, including trauma, cardiac arrest, severe sepsis and septic shock. ${ }^{3-6}$

Single measurement of lactate cannot improve clinical outcome, but therapeutic strategies that can potentially decrease arterial lactate may be associated with improved clinical outcomes. The aim of such a strategy is to reverse the global tissue hypoxia. The idea of lactate clearance (LC) is to accommodate this concept. Many clinical studies have demonstrated that patients with LC showed better clinical outcomes as compared with those without LC; furthermore, patients with rapid LC were more likely to survive than those with slow LC. ${ }^{7-11}$ However, the diagnostic performance of LC in predicting mortality has not been well defined and conflicting results exist among studies. For instance, the area under receiver operating characteristic curve (AU-ROC) ranged from 0.67 to 0.87 as reported by different studies. We here presented a study protocol for a systematic review to investigate the association of LC with clinical outcomes. 


\section{METHODS}

\section{Inclusion criteria of studies}

Participants

Critically ill adult patients will be included in our analysis. This population will include heterogeneous study participants, including patients with sepsis or severe sepsis, trauma, surgical intensive care unit (ICU) patients and so on. Children will be excluded because lactate metabolism in children is different. ${ }^{12}$

\section{Exposed group and control group}

Patients with LC are used as the exposed group. LC will be defined from two aspects: magnitude and time needed for the clearance. A threshold of $10 \%$ reduction from baseline or normalisation is employed, and 24 hour is used as the time frame for LC. The control group is defined as those without LC.

\section{Study endpoints}

The primary outcome is mortality. The pooled data will show how the risk of death differs between patients with and without LC. Furthermore, the diagnostic performance of LG in predicting mortality will be reported. Statistics for diagnostic performance including sensitivity, specificity, positive likelihood ratio and negative likelihood ratio will be reported.

\section{Studies to be included}

Both observational cohort studies and randomised controlled trials (RCT) will be included for analysis. Observational studies will include both prospective and retrospective studies.
Search methods for identifying studies

We will search four databases including EBSCO, PubMed, Scopus and ISI Web of knowledge from inception to February 2014. There will be no language restrictions in the electronic search for studies.

The searched item will include terms related to critically ill patients (intensive care, ICU, critically ill, intensive care unit, critical care, severely ill and emergency) and terms related to LC (lactate clearance, lactate normalisation, kinetics and lactic clearance). The specific searching strategies for the four databases are shown in table 1. References of all relevant articles will be searched by hand to uncover other potentially eligible studies. If necessary, experts of this field will be contacted for additional studies for our topic.

\section{Data collection}

A standardised form will be made for data abstraction. The following data will be extracted from original articles: the name of the first author, publication year, male percentage, subjects (ie, sepsis, major surgery) and setting (ie, medical ICU, surgical ICU, or emergency department), mean age of study population, sample size, mortality, definition of clearance (ie, the percentage reduction and time frame for LC) and the initial lactate level. Data used for meta-analysis will be as follows: the numbers of survivors and non-survivors in the LC group and the non-LC groups; the AU-ROCs curve and the corresponding confidence interval; the cut-off value of LC for the best sensitivity and specificity to predict mortality. Other secondary outcomes will be included if available in original articles. If these data were unavailable in original articles, we tried to contact the corresponding authors for more data.

Table 1 Searching strategy in PubMed, EBSCO, ISI Web of knowledge and Scopus

\begin{tabular}{|c|c|c|c|}
\hline PubMed & EBSCO & ISI Web of knowledge & Scopus \\
\hline \#1 lactate clearance[Title/ & S1: AB lactate clearance & \#1 Topic=('lactate clearance') & \#1 (TITLE-ABS-KEY('lactate \\
\hline Abstract] OR lactate & OR AB lactate normalization & OR Topic $=$ ('lactate & clearance') OR \\
\hline normalization[Title/Abstract] & OR AB lactate kinetics OR & normalization') OR Topic= & TITLE-ABS-KEY('lactate \\
\hline OR lactate kinetics[Title/ & $A B$ lactic clearance $O R A B$ & ('lactate kinetics') OR Topic= & normalization') OR \\
\hline Abstract] OR lactic & hyperlactatemia & ('lactic clearace') OR Topic= & TITLE-ABS-KEY('lactate \\
\hline clearance[Title/Abstract] OR & & ('hyperlactatemia') & kinetics') OR TITLE-ABS-KEY \\
\hline hyperlactatemia[Title/ & & Timespan=All years Search & ('lactic clearance') OR \\
\hline Abstract] & & language =Auto & $\begin{array}{l}\text { TITLE-ABS-KEY } \\
\text { ('hyperlactatemia')) }\end{array}$ \\
\hline \#2 (intensive care[Title/ & S2: AB intensive care OR & \#2 Topic=('intensive care') OR & \#2 (TITLE-ABS-KEY('intensive \\
\hline Abstract] OR ICU[Title/ & AB ICU OR AB critically ill & Topic=('ICU') OR Topic= & care') OR TITLE-ABS-KEY \\
\hline Abstract] OR critically ill & OR $A B$ intensive care unit & ('critically ill') OR Topic= & ('ICU') OR TITLE-ABS-KEY \\
\hline [Title/Abstract] OR intensive & OR $A B$ critical care OR $A B$ & ('intensive care unit') OR & ('critically ill') OR \\
\hline care unit[Title/Abstract] OR & severely ill OR $A B$ & Topic=('critical care') OR & TITLE-ABS-KEY('intensive \\
\hline critical care[Title/Abstract] & emergency & Topic=('severely ill') OR & care unit') OR TITLE-ABS-KEY \\
\hline OR severely ill[Title/ & & Topic=('emergency') & ('critical care') OR \\
\hline Abstract] OR emergency & & Timespan=Al/ years & TITLE-ABS-KEY('severely ill') \\
\hline [Title/Abstract]) & & Search language=Auto & $\begin{array}{l}\text { OR TITLE-ABS-KEY } \\
\text { ('emergency')) }\end{array}$ \\
\hline \#1 AND \#2 & S1 AND S2 & \#1 AND \#2 & \#1 AND \#2 \\
\hline
\end{tabular}




\section{Quality assessment of included studies}

Newcastle Ottawa Scale for cohort study will be employed to assess the reporting quality of included original studies. ${ }^{13}$ This scale comprises eight items evaluating the quality of observational cohort studies in terms of selection, comparability and outcome. If patients were not followed up for the entire length of stay in hospital (eg, ICU mortality), the follow-up was thought to be not long enough for the outcome to occur. Table 2 shows the criteria used for quality assessment adapted from Newcastle Ottawa Scale.

\section{Data synthesis}

Mantel-Haenszel methods will be used to combine effect size for fixed-effect model. Otherwise, if there are significant heterogeneity in component studies, the random-effects method will be used by assuming that the different studies are estimating different effects. This method is based on the inverse variance approach, assigning study weights according to variance. ${ }^{14}$ We will report relative risk (RR) of death for those with LC and those without, with an $\mathrm{RR}<1$ indicating $\mathrm{LC}$ is associated with a lower risk of death and a RR $>1$ indicating $L C$ is a risk factor for death. For continuous outcome variables, the mean difference will be used to measure effect size. Standard deviations together with sample size will be used to assign weight to each study. For variables in difference scale, standardised mean difference will be used, which standardises the mean difference by SD to a single scale.

The diagnostic performance of LC in predicting mortality will be of interest to clinicians; thus, we will synthesize data regarding diagnostic statistics. Owing to expected substantial between-study heterogeneity, we will employ the hierarchical summary receiver operating characteristic model. This model assumes that there is an underlying ROC curve in each study with parameters $\alpha$ and $\beta$ that represent the accuracy and asymmetry of the ROC curve. ${ }^{15}$ The reported pooled statistics include sensitivity, specificity, positive likelihood ratio and negative likelihood ratio. Data synthesis will be performed by using the software Stata (College Station, Texas, USA).

\section{Heterogeneity}

Heterogeneity will be assessed by using a statistical test. Inconsistency across component studies will be quantified by using the equation:

$$
\mathrm{I}^{2}=\left(\frac{\mathrm{Q}-\mathrm{df}}{\mathrm{Q}}\right) \times 100 \%
$$

where $\mathrm{Q}$ is the $\chi^{2}$ statistic and $\mathrm{df}$ is its degree of freedom. ${ }^{16} 17 \mathrm{I}^{2}$ ranges between $0 \%$ and $100 \%$, with $0-40 \%$ indicating no important heterogeneity, 40-60\% indicating moderate heterogeneity, $60-90 \%$ indicating substantial heterogeneity, and $>90 \%$ indicating considerable heterogeneity. Heterogeneity will be explored by using subgroup analysis restricting to sepsis or septic shock. Depending on the number of studies available, other subgroup analyses involving trauma, major operation will be explored. Sensitivity analysis will be performed by excluding retrospective studies.

\section{Publication bias}

The contour enhanced funnel plot will be used to detect publication bias. RR from each study is plotted against their variance. Asymmetrical appearance of the plot indicates the presence of publication bias. Contours of statistical significance are overlaid on the funnel plot, which facilitates the assessment of whether the areas where component studies locate are areas of statistical significance and whether the areas where studies are potentially missing are areas of low significance. In situations when studies are missing in areas of low significance, it is possible that the asymmetry is due to publication bias. Otherwise, if the areas where studies are missing are areas of high statistical significance, publication bias is less likely to be present. ${ }^{18}$ Egger's test will

\begin{tabular}{|c|c|c|}
\hline \multirow[t]{4}{*}{ Selection } & $\begin{array}{l}\text { Representativeness of the exposed } \\
\text { cohort }\end{array}$ & $\begin{array}{l}\text { This item will be assigned a ' } \star \text { ' when all eligible patients with LC are } \\
\text { included in analysis during study period }\end{array}$ \\
\hline & Selection of the non-exposed cohort & $\begin{array}{l}\text { This item will be assigned a ' } \star \text { 'when all eligible patients with non-LC } \\
\text { are included in analysis during study period }\end{array}$ \\
\hline & Ascertainment of exposure & $\begin{array}{l}\text { This item will be assigned a ' } \star \text { ' when lactate is directly obtained from } \\
\text { medical chart, not by reporting from the patient }\end{array}$ \\
\hline & $\begin{array}{l}\text { Outcome of interest is not present at } \\
\text { the start of study }\end{array}$ & $\begin{array}{l}\text { This item will be assigned a ' } \star \text { ' when death is not occurred within } \\
\text { the time frame for determining LC }\end{array}$ \\
\hline Comparability & $\begin{array}{l}\text { Comparability of cohorts on the basis } \\
\text { of design or analysis }\end{array}$ & $\begin{array}{l}\text { Baseline characteristics of LC and non-LC patients are comparable. } \\
\text { Usually this can be found in table } 1\end{array}$ \\
\hline \multirow[t]{3}{*}{ Outcome } & Assessment of outcome & $\begin{array}{l}\text { This item will be assigned a ' } \star \text { ' when mortality is assessed by } \\
\text { investigator, not by the report of the patient's family or next-of-kin. }\end{array}$ \\
\hline & $\begin{array}{l}\text { Is follow-up long enough for outcome } \\
\text { to occur }\end{array}$ & The adequate follow-up is during hospital stay \\
\hline & Adequacy of follow-up of cohort & This item will be assigned a ' $\star$ ' when follow-up rate $>95 \%$ \\
\hline
\end{tabular}


be used to test the asymmetry of the funnel plot. It is tested by the linear regression of RR against its standard, weighted by the inverse variance of RR. ${ }^{19}$

\section{DISCUSSION}

LC has been incorporated into the initial resuscitation target in the 2012 Surviving Sepsis Campaign guideline. ${ }^{20}$ It is stated "We suggest targeting resuscitation to normalize lactate in patients with elevated lactate levels as a marker of tissue hypoperfusion." This is based on empirical evidence that hyperlactatemia is associated with significantly increased risk of death. Furthermore, two multicentre trials evaluated the resuscitation strategy that included LC as a target. The result showed that quantitative resuscitation based on LC was non-inferior to that based on central venous oxygenation saturation. ${ }^{21}$ The other showed that the resuscitation strategy based on both LC and central venous oxygenation saturation was associated with a $9.6 \%$ absolute reduction in mortality. ${ }^{22}$ The results of these two RCTs are encouraging and inspire more researches on this field. Many studies have been carried out aiming to investigate the association of LC with mortality. ${ }^{711}$ These studies reported conflicting results. For instance, the sensitivity of LC in predicting mortality ranged from 0.45 to 0.97 ; and the specificity ranged from 0.52 to 0.84 . Such conflicting results may confuse clinicians on whether LC can be utilised for clinical decision-making, or in what situation can LC provide the most useful information. Therefore, a systematic review is mandatory at this stage to clarify this linkage. Subgroup analysis restricted to patients with sepsis will provide further insights into the clinical usefulness of LC.

Contributors ZZ and KC made substantial contributions to conception and design, acquisition of data, or analysis and interpretation of data; XX helped in drafting the article and revising it critically for important intellectual content; and all authors gave final approval of the version to be published.

Competing interests None.

Provenance and peer review Not commissioned; externally peer reviewed.

Open Access This is an Open Access article distributed in accordance with the Creative Commons Attribution Non Commercial (CC BY-NC 3.0) license, which permits others to distribute, remix, adapt, build upon this work noncommercially, and license their derivative works on different terms, provided the original work is properly cited and the use is non-commercial. See: http:// creativecommons.org/licenses/by-nc/3.0/

\section{REFERENCES}

1. Fall PJ, Szerlip HM. Lactic acidosis: from sour milk to septic shock. J Intensive Care Med 2005;20:255-71.

2. Okorie ON, Dellinger P. Lactate: biomarker and potential therapeutic target. Crit Care Clin 2011;27:299-326.

3. Trzeciak S, Dellinger RP, Chansky ME, et al. Serum lactate as a predictor of mortality in patients with infection. Intensive Care Med 2007;33:970-7.

4. Kliegel A, Losert H, Sterz F, et al. Serial lactate determinations for prediction of outcome after cardiac arrest. Medicine 2004;83:274-9.

5. Abramson D, Scalea T, Hitchcock R, et al. Lactate clearance and survival following injury. J Trauma 1993;35:584-9.

6. Blow O, Magliore L, Claridge JA, et al. The golden hour and the silver day: detection and correction of occult hypoperfusion within 24 hours improves outcome from major trauma. J Trauma 1999;47:964-9.

7. Nguyen HB, Rivers EP, Knoblich BP, et al. Early lactate clearance is associated with improved outcome in severe sepsis and septic shock. Crit Care Med 2004;32:1637-42.

8. Jansen TC, van Bommel J, Schoonderbeek FJ, et al. Early lactate-guided therapy in intensive care unit patients: a multicenter, open-label, randomized controlled trial. Am J Respir Crit Care Med 2010;182:752-61.

9. Arnold RC, Shapiro NI, Jones AE, et al. Multi-center study of early lactate clearance as a determinant of survival in patients with presumed sepsis. Shock 2009;32:35-9.

10. McNelis J, Marini C, Jurkiewicz A, et al. Prolonged lactate clearance is associated with increased mortality in the surgical intensive care unit. Am J Surg 2001;182:481-5.

11. Bakker J, Gris P, Coffernils $P$, et al. Serial blood lactate levels can predict the development of multiple organ failure following septic shock. Am J Surg 1996;171:221-6.

12. Zafeiridis A, Dalamitros A, Dipla K, et al. Recovery during high-intensity intermittent anaerobic exercise in boys, teens, and men. Med Sci Sports Exerc 2005;37:505-12.

13. Wells G, Shea B, O'Connell J, et al. The Newcastle-Ottawa scale (NOS) for assessing the quality of nonrandomised studies in meta-analysis. http://www.ohri.ca/programs/clinical_epidemiology/ oxfordasp

14. DerSimonian R, Laird N. Meta-analysis in clinical trials. Control Clin Trials 1986;7:177-88.

15. Rutter CM, Gatsonis CA. A hierarchical regression approach to meta-analysis of diagnostic test accuracy evaluations. Stat Med 2001;20:2865-84.

16. Higgins JPT, Thompson SG, Deeks JJ, et al. Measuring inconsistency in meta-analyses. BMJ 2003;327:557-60.

17. Higgins JPT, Thompson SG. Quantifying heterogeneity in a meta-analysis. Stat Med 2002;21:1539-58.

18. Peters JL, Sutton AJ, Jones DR, et al. Contour-enhanced meta-analysis funnel plots help distinguish publication bias from other causes of asymmetry. J Clin Epidemiol 2008;61:991-6.

19. Egger M, Smith GD, Schneider M, et al. Bias in meta-analysis detected by a simple, graphical test. BMJ 1997;315:629-34.

20. Dellinger RP, Levy MM, Rhodes A, et al. Surviving Sepsis Campaign Guidelines Committee including The Pediatric Subgroup. Surviving Sepsis Campaign: international guidelines for management of severe sepsis and septic shock, 2012. Intensive Care Med 2013;39:165-228.

21. Jones AE, Shapiro NI, Trzeciak S, et al. Emergency Medicine Shock Research Network (EMShockNet) Investigators. Lactate clearance vs central venous oxygen saturation as goals of early sepsis therapy: a randomized clinical trial. JAMA 2010;303:739-46.

22. Jansen TC, van Bommel J, Schoonderbeek FJ, et al. LACTATE study group. Early lactate-guided therapy in intensive care unit patients: a multicenter, open-label, randomized controlled trial. Am J Respir Crit Care Med 2010;182:752-61. 